\title{
Trajectories of rotations
}

\author{
by \\ Pierre Arnoux, Sébastien Ferenczi and \\ Pascal Hubert (Marseille)
}

Among the fundamental sequences in arithmetics, symbolic dynamics and language theory are the Sturmian sequences, which are the non-periodic sequences with the smallest number of factors; they have been studied extensively since [HED-MOR], see for example the survey in [ALL]. They are "canonical" codings of the trajectories of irrational rotations on the one-dimensional torus (see definition of $U(\alpha, x)$ below) and this coding is intrinsically linked to the continued fraction approximation of the argument.

The explicit determination of this coding is "in the folklore" for the point $x=0$; for other points, although the method is easy in principle, technicalities have to be overcome to get manageable formulas. An algorithm is given in [ITO-YAS] and another can be deduced from [RAU1], [RAU2], but they do not use the standard continued fraction approximation. A closely related process is studied in detail in [SID-VER], using the notion of adic systems; in that paper two different kinds of approximation are shown, dual to each other; we shall come back to this topic from the point of view of symbolic dynamics at the end of the present paper. [ARN-FIS] gives a general overview of the subject, relating symbolic dynamics, arithmetic and some geometric models. All these approaches use the dynamical notion of induction, which became famous as the Rauzy induction ([RAU3]). Another technique, using formal power series, was used in [NIS-SHI-TAM]; this paper was later corrected by Komatsu, and his papers ([KOM1]-[KOM5]) give the only known complete characterization of these sequences which is linked to the canonical continued fraction approximation.

The following construction gives a conceptual characterization of the Sturmian sequence $U(\alpha, x)$ for every given $\alpha$ and $x$; the proof and the actual expression of the sequence are somewhat simpler than in Komatsu's main result (Theorem 3 of [KOM2]), while they use both the standard con-

1991 Mathematics Subject Classification: 11J70. 
tinued fraction approximation and a version of Rauzy induction, and thus are guided by the dynamics of the system.

Definition 1. Let $0<\alpha<1$ be an irrational number and $T=T_{\alpha}$ be the rotation on $\mathbb{R} / \mathbb{Z}$ defined by $T_{\alpha} y=y+\alpha \bmod 1$. Let $P_{0}$ be the set $\left[0,1-\alpha\left[\right.\right.$ and $P_{1}$ the set $[1-\alpha, 1[$; let $x$ be a point in $[0,1[$. The Sturmian coding of the rotation $T_{\alpha}$, denoted by $U(\alpha, x)$, is the sequence $u_{n}, n \geq 0$, defined by $u_{n}=0$ whenever $T_{\alpha}^{n} x \in P_{0}, u_{n}=1$ otherwise.

Our main result is the following theorem:

TheOREM 1. Let

$$
\alpha=\left[0 ; a_{1}+1, a_{2}, \ldots, a_{n}, \ldots\right], \quad \alpha_{n}=\left[0 ; a_{n}, \ldots\right],
$$

with the usual notation for the standard continued fraction approximation; let $x_{1}=\frac{x}{1-\alpha}-1$, and then

$$
\begin{gathered}
x_{n+1}=-\frac{x_{n}}{\alpha_{n}}-e_{n}, \quad y_{n}=\frac{x_{n}}{\alpha_{n}}+\alpha_{n+1}, \\
e_{n}= \begin{cases}\sup \left(-\left[y_{n}\right], 0\right) & \text { if } y_{n} \text { is not an integer, } \\
\sup \left(-y_{n}, 0\right) & \text { if } y_{n} \text { is an integer and } n \text { is odd, } \\
\sup \left(-y_{n}+1,0\right) & \text { if } y_{n} \text { is an integer and } n \text { is even. }\end{cases}
\end{gathered}
$$

Let

$$
W_{n+1}=W_{n-1} W_{n}^{a_{n+1}}, \quad Z_{n+1}=Z_{n} W_{n}^{e_{n+1}}
$$

for all $n$, with $W_{0}=0, W_{1}=10^{a_{1}}, Z_{1}=0^{e_{1}}$ and the convention $a^{0}=\emptyset$. Then

$$
U(\alpha, x)=\lim _{n \rightarrow \infty} Z_{n}
$$

when the $e_{n}$ are not ultimately zero,

$$
U(\alpha, x)=\lim _{n \rightarrow \infty} Z_{N-1} W_{2 n+1}
$$

when $e_{n}=0$ for every $n \geq N \geq 2$, and

$$
U(\alpha, x)=\lim _{n \rightarrow \infty} W_{2 n+1}
$$

when $e_{n}=0$ for every $n \geq 1$, which corresponds to the case $x=1-\alpha$.

The digits $e_{n}$ have a nice arithmetic interpretation: this is the wellknown algorithm of Ostrowski [OST], which gives an approximation of $x$ by multiples of $\alpha$, and was used for example in [SOS], [KEA] or [SID-VER]. We have the following relation:

Proposition 1.

$$
x=1+(1-\alpha)\left(-e_{1}+\sum_{n=1}^{\infty}(-1)^{n+1} \alpha_{1} \ldots \alpha_{n} e_{n+1}\right) .
$$




\section{Preliminaries}

Definition 2. A Sturmian sequence is a sequence on the finite alphabet $\{0,1\}$ which contains exactly $n+1$ different factors of length $n$.

Let $T$ be as defined above; by abuse of notation, we shall identify it with the same transformation on the fundamental domain $\left[0,1\left[\right.\right.$; then $T_{\alpha}$ is an exchange of two intervals, with one discontinuity, at the point $1-\alpha$. Hence the sequence $U(\alpha, x)$ defined above is a natural coding in this setting; this sequence is also called the positive P-name of the point $x$ under the transformation $T_{\alpha}$.

We check that this sequence is also defined by $u_{n}=[(n+1) \alpha+x]-[n \alpha+x]$ for any $n \geq 0$. It is proved in [HED-MOR] that any Sturmian sequence is either some $U(\alpha, x)$, for an irrational $\alpha$, or the similar quantity obtained by taking the intervals open on the left and closed on the right. This last quantity can be computed by the same reasoning as in Theorem 1, with the necessary changes in the definition of $e_{n}$.

2. The symmetric Rauzy induction. We suppose first that $\alpha<1 / 2$. We begin by a renormalization so that the picture will be more symmetric with respect to the point $1-\alpha$; at the same time, to make computations a little simpler, we make the length of one of the intervals equal to one: we apply to every point the affine map $y \rightarrow \frac{y}{1-\alpha}-1$. Let $x_{1}=\frac{x}{1-\alpha}-1$ and $\alpha_{1}=\frac{\alpha}{1-\alpha}$. The rotation $T_{\alpha}$ becomes the following exchange of two intervals (see Figure 1), denoted by $T_{1}$ :

$$
T_{1} y= \begin{cases}y+\alpha_{1} & \text { if } y \in P_{0}^{1}=P_{0}=[-1,0[, \\ y-1 & \text { if } y \in P_{1}^{1}=P_{1}=\left[0, \alpha_{1}[.\right.\end{cases}
$$

The positive $P$ - or $P^{1}$-name of $x_{1}$ under $T_{1}$ is

$$
V\left(x_{1}, T_{1}\right)=U(\alpha, x) .
$$

Let $J$ be the interval $\left[-\alpha_{2} \alpha_{1}, \alpha_{1}\left[\right.\right.$, where $-\alpha_{2} \alpha_{1}=-1+a_{1} \alpha_{1}, a_{1}=\left[1 / \alpha_{1}\right]$ and $\alpha_{2}=\left\{1 / \alpha_{1}\right\}$ ( $\alpha_{1}$ is irrational as $\alpha$ is, and is in ]0,1[).

As $x_{1}$ is not necessarily in $J$, let $e_{1}$ be the smallest natural integer $e$ such that $x_{1}+e \alpha_{1} \in J$; set $x_{1}^{\prime}=x_{1}+e_{1} \alpha_{1}$. So we have $x_{1}^{\prime}=T_{1}^{e_{1}} x_{1}$, and, if $e_{1}$ is not zero, then $x_{1}, T_{1} x_{1}, \ldots, T_{1}^{e_{1}-1} x_{1}$ must be in $\left[-1, \alpha_{1}\left[\backslash J\right.\right.$, hence in $P_{0}$; hence the $P$-name of $x_{1}$ is a string of $e_{1}$ zeros followed by the $P$-name of $T_{1}^{e_{1}} x_{1}$, so

$$
V\left(x_{1}, T_{1}\right)=0^{e_{1}} V\left(x_{1}^{\prime}, T_{1}\right) .
$$

If $x<0$ then $e_{1}$ is the smallest integer such that $x+e_{1} \alpha_{1} \geq-\alpha_{1} \alpha_{2}$, while if $x \geq 0$ then $e_{1}=0$, hence $e_{1}$ is the maximum of $\left[-x / \alpha_{1}-\alpha_{2}\right]+1$ and 0 , except that the first argument must be replaced by $\left[-x / \alpha_{1}-\alpha_{2}\right]$ if it is an integer. Note that $0 \leq e_{1} \leq\left[1 / \alpha_{1}\right]=a_{1}$. 


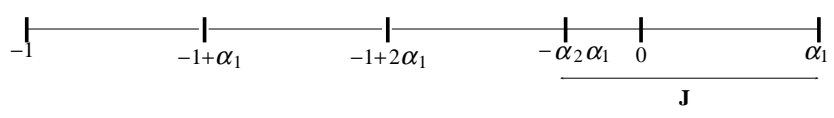

Fig. 1. Rauzy induction in the case $a_{1}=3$

We now make what is called the symmetric Rauzy induction:

Lemma 1. For a point $y$ in $J$, let $t(y)$ be the first return time of $y$ to $J$, and $R y=T_{1}^{t(y)} y$ the induced map of $T_{1}$ on $J$. Put $Q_{0}=\left[-1+a_{1} \alpha_{1}, 0[\right.$, and $Q_{1}=\left[0, \alpha_{1}\left[\right.\right.$. For $y=x_{1}^{\prime}$, let $u$ be the sequence $V\left(y, T_{1}\right)$ and $v$ be the positive $Q$-name of $y$ under $R$. Define the substitution $\sigma_{a_{1}}$ by $\sigma_{a_{1}} 0=0$ and $\sigma_{a_{1}} 1=10^{a_{1}}$. Then

$$
u=\sigma_{a_{1}} v
$$

Proof. We compute $t$ and $R$ :

- if $y$ is in $Q_{0}$, then $T_{1} y$ is in $\left[-1+\left(a_{1}+1\right) \alpha_{1}, \alpha_{1}\left[\subset\left[0, \alpha_{1}[\subset J\right.\right.\right.$ and so $t(y)=1$ and $R y=y+\alpha_{1}$;

- if $y$ is in $Q_{1}$, then $T_{1}^{l} y$ is in $\left[-1+(l-1) \alpha_{1},-1+l \alpha_{1}\left[\subset\left[-1, a_{1} \alpha_{1}-1[\subset\right.\right.\right.$ $\left[-1, \alpha_{1}\left[\backslash J\right.\right.$ for $1 \leq l \leq a_{1}$, then $T_{1}^{a_{1}+1} y$ is in $\left[-1+a_{1} \alpha_{1},-1+\left(a_{1}+1\right) \alpha_{1}[\subset J\right.$; hence $t(y)=a_{1}+1$ and $R y=y+a_{1} \alpha_{1}-1$.

Suppose we know $v$ and we want to find $u$; $T_{1}^{n} y$ will be in $J$ for $n=0$, $n=t(y), n=t_{2}(y)=t(y)+t(R y)$, the second return time of $y$ to $J, \ldots, n=$ $t_{k}(y)=t_{k-1}(y)+t\left(R^{k-1} y\right)$, which are the return times of $y$ to $J$ for all positive $k$. Moreover,

- if $v_{k}=0$, then $R^{k} y=T_{1}^{t_{k}(y)} y$ is in $Q_{0} \subset P_{0}$, and, by the last paragraph, $t\left(R^{k} y\right)=1$ and hence $t_{k+1}(y)=t_{k}(y)+1$;

- if $v_{k}=1$, then $R^{k} y=T_{1}^{t_{k}(y)} y$ is in $Q_{1} \subset P_{1}$, and so, again by the last paragraph, $T_{1}^{t_{k}(y)+l} y$ is in $\left[-1, a_{1} \alpha_{1}-1\left[\subset P_{0}\right.\right.$ for $1 \leq l \leq a_{1}$, and $t\left(R^{k} y\right)=a_{1}$, and so $t_{k+1}(y)=t_{k}(y)+a_{1}$.

From this analysis, it results that $u_{t_{k}(y)}=v_{k}$ for all $k$; when two consecutive $t_{k}(y)$ differ by more than 1 , then they differ by $a_{1}+1, v_{k}=1$, and the digits $u_{t_{k}(y)+1}, \ldots, u_{t_{k}(y)+a_{1}}$ are all equal to 0 . For short, we say that $u$ is deduced from $v$ by the substitution $\sigma_{a_{1}}$ defined above.

\section{Symbolic dynamics of trajectories}

LEMma 2. With the notations of Theorem 1 , if $\tau 0=1, \tau 1=0$, and

$$
\begin{aligned}
& T_{n} y=\left\{\begin{array}{ll}
y+\alpha_{n} & \text { if } y \in P_{0}^{n}=[-1,0[, \\
y-1 & \text { if } y \in P_{1}^{n}=\left[0, \alpha_{n}[\right.
\end{array} \quad \text { if } n\right. \text { is odd, } \\
& T_{n} y=\left\{\begin{array}{ll}
y+\alpha_{n} & \text { if } \left.\left.y \in P_{0}^{n}=\right]-1,0\right], \\
y-1 & \text { if } \left.y \in P_{1}^{n}=\right] 0, \alpha_{n}
\end{array}\right]
\end{aligned}
$$


then

$$
V\left(x_{n}, T_{n}\right)=0^{e_{n}} \sigma_{a_{n}} \tau V\left(x_{n+1}, T_{n+1}\right) .
$$

Proof. Now we consider the mapping $R$ on $J$, and apply to every point the affine map $y \rightarrow-y / \alpha_{1}$. Then, applying this mapping to the expression of $R$ we have found, we see that $R$ becomes the map $T_{2}$, defined above. As $Q_{0}$ becomes $P_{1}^{2}$ and $Q_{1}$ becomes $P_{0}^{2}$, the $Q$-names under $R$ are the images of the $P^{2}$-names under $T_{2}$ by the map $\tau$. Note that $\alpha_{2}$ is still irrational. So we get

$$
v=\tau V\left(x_{2}, T_{2}\right), \quad \text { where } \quad x_{2}=-x_{1}^{\prime} / \alpha_{1} .
$$

We can now iterate this process: we repeat the previous construction with $T_{1}, \alpha_{1}, x_{1}$ replaced by $T_{2}, \alpha_{2}, x_{2}$ and all semi-open intervals $[a, b[$ replaced by $] a, b]$, and vice-versa; this gives us $a_{2}$ as in the previous case, and the induction interval will be $\left.]-1+a_{2} \alpha_{2}, \alpha_{2}\right] ; e_{2}$ will again be the smallest integer $e$ such that $x_{2}+e \alpha_{2}$ falls into the induction interval; the change in the boundaries gives a slightly different exact value of $e_{2}$, as the formula with the integral part plus one is now valid all the time; then we define $x_{2}^{\prime}$, $\alpha_{3}, x_{3}$, and $T_{3}$ which is defined like $T_{1}$, with semi-open intervals of the form $[a, b[$. So we have, for $n \geq 1$, the recursion formula we claim, with

$$
\left(\alpha_{n+1}, x_{n+1}\right)=\left(\frac{1}{\alpha_{n}}-a_{n},-\frac{x_{n}}{\alpha_{n}}-e_{n}\right), \quad a_{n}=\left[\frac{1}{\alpha_{n}}\right],
$$

and the $e_{n}$ as defined in Theorem 1. We check that these formulas define the $a_{n}$ and $\alpha_{n}$ as stated in Theorem 1 .

\section{Arithmetic and combinatorics}

LEMMA 3. Let $b_{n}=0$ and $i_{n}=1$ for $n$ even, $b_{n}=1$ and $i_{n}=0$ for $n$ odd, $\sigma_{0} 0=0, \sigma_{0} 1=10, \sigma_{1} 0=01, \sigma_{1} 1=1$. If the sequence $e_{n}$ is not ultimately identical to zero, then

$$
U(\alpha, x)=\lim _{n \rightarrow \infty} 0^{e_{1}} \sigma_{0}^{a_{1}}\left(1^{e_{2}}\right) \ldots\left(\sigma_{0}^{a_{1}} \ldots \sigma_{i_{n}}^{a_{n}}\right)\left(b_{n}^{e_{n+1}}\right) .
$$

Proof. As the $a_{n}$ are all strictly positive, the recursion formulas allow us, if we know the first letter of $V\left(x_{n+2}, T_{n+2}\right)$, to know at least two letters of $V\left(x_{n}, T_{n}\right)$, and hence at least $2^{n / 2}$ letters of $V\left(x_{1}, T_{1}\right)$. Hence the result.

LEMMA 4. With the same notations as before, if $e_{n}=0$ for every $n \geq N$, then

$$
\begin{aligned}
& U(\alpha, x) \\
& =0^{e_{1}} \sigma_{0}^{a_{1}}\left(1^{e_{2}}\right) \ldots\left(\sigma_{0}^{a_{1}} \ldots \sigma_{i_{N-2}}^{a_{N-2}}\right)\left(b_{N}^{e_{N-1}}\right)\left(\sigma_{0}^{a_{1}} \ldots \sigma_{i_{N-2}}^{a_{N-2}} \sigma_{i_{N-1}}^{a_{N-1}} V\left(0, T_{N}\right)\right), \\
& V\left(0, T_{N}\right)=\lim _{n \rightarrow \infty}\left(\sigma_{i_{N}}^{a_{N}} \ldots \sigma_{i_{2 n+1}}^{a_{2 n+1}}\right)(0) .
\end{aligned}
$$


Proof. Suppose that $e_{n}=0$ for every $n \geq N$. This is the case if and only if $x$ belongs to the non-positive orbit of 0 ; this is the only moment where we need to know that the intervals are open on the left, as there could be two possible codings; in this case, for some $i \geq 0$ independent of $n, u_{i} \ldots u_{i+n-1}$ is the unique factor of length $n$ which has two extensions on the left. Then $x_{n+1}=-x_{n} / \alpha_{n}$, and $x_{n}$ is in the induction interval, for all $n \geq N$; this ensures that $\left|x_{N}\right| \leq \alpha_{N} \ldots \alpha_{N+n}$ for $n$ arbitrarily large, hence $x_{N}=0$, and so $x_{n}=0$ for every $n \geq N$. Moreover, the first digit of $V\left(0, T_{n}\right)$ is zero for $n$ even and is one for $n$ odd.

Proof of Theorem 1. We reformulate our results, in a more constructive way; with the notations of Lemma 3 , let $W_{n}$ be the word

$$
\sigma_{0}^{a_{1}} \ldots \sigma_{i_{n}}^{a_{n}} b_{n}
$$

for $n \geq 1$. Let $Z_{n}$ be the word

$$
0^{e_{1}} \sigma_{0}^{a_{1}}\left(1^{e_{2}}\right) \ldots\left(\sigma_{0}^{a_{1}} \ldots \sigma_{i_{n-1}}^{a_{n-1}}\right)\left(b_{n-1}^{e_{n}}\right)
$$

for $n \geq 2, Z_{1}$ being the word $0^{e_{1}}$. Then $Z_{n+1}=Z_{n} W_{n}^{e_{n+1}}$. The recursion giving the $W_{n}$ has to be made precise a little. Let $U_{n}$ be $\sigma_{0}^{a_{1}} \ldots \sigma_{i_{n}}^{a_{n}}(0)$ and $V_{n}$ be $\sigma_{0}^{a_{1}} \ldots \sigma_{i_{n}}^{a_{n}}(1)$. Then we have

$$
\begin{array}{ll}
U_{2 n+1}=U_{2 n}=W_{2 n}, & V_{2 n+2}=V_{2 n+1}=W_{2 n+1}, \\
V_{2 n+1}=V_{2 n} U_{2 n}^{a_{2 n+1}}, & U_{2 n+2}=U_{2 n+1} V_{2 n+1}^{a_{2 n+2}} .
\end{array}
$$

This proves the theorem for the case $\alpha<1 / 2$.

If $\alpha>1 / 2$, we can apply the same algorithm, except that at the beginning $a_{1}=e_{1}=0$ and $\alpha_{1}>1$; the first induction is then trivial as $J=\left[-1, \alpha_{1}\left[\right.\right.$, and, after the renormalization $y \rightarrow-y / \alpha_{1}$, we are in the same situation as when we have started from an $\alpha<1 / 2$; hence Theorem 1 is proved in that case also.

Some particular cases. As our method put $x=1-\alpha$ in the centre of the picture, there is an explicit formula for $U(\alpha, 1-\alpha)$ (see the last formula in Theorem 1). To get $U(\alpha, 0)$, the easiest way is to use the fact that $0=T(1-\alpha)$, hence

$$
U(\alpha, 0)=S U(\alpha, 1-\alpha),
$$

where $S\left(u_{0} u_{1} u_{2} \ldots\right)=u_{1} u_{2} \ldots$

If we choose $\alpha=\phi=(\sqrt{5}-1) / 2$, then, with our notations, $a_{1}=0$ and $a_{n}=1$ for every $n \geq 2$. The famous Fibonacci sequence $F$, defined as the fixed point of the substitution $0 \rightarrow 01,1 \rightarrow 0$, is in fact $\tau U(\phi, \phi)$; hence our algorithm gives $F=S^{2} F^{\prime}$ with $F^{\prime}=\lim _{n \rightarrow \infty} W_{2 n+1}, W_{0}=1, W_{1}=0$, $W_{n+1}=W_{n-1} W_{n}$, which is a non-standard way to build that sequence, but we can check that the two constructions give the same sequence. 
Proof of Proposition 1. We may write $x_{n}=-\alpha_{n} x_{n+1}-e_{n}$, hence $x_{1}=$ $-e_{1}+\sum_{i=1}^{n-1}(-1)^{i+1} \alpha_{1} \ldots \alpha_{i} e_{i+1}+(-1)^{n} \alpha_{1} \ldots \alpha_{n} x_{n+1}$; now, it is well known that, as $a_{n} \alpha_{n}+\alpha_{n} \alpha_{n+1}=1$, and $\alpha_{n+1} \leq 1$, we have $\alpha_{n} \alpha_{n+1} \leq 1 / 2$; hence we get the assertion of the proposition.

It is well known that the product $\alpha_{1} \ldots \alpha_{n}$ is closely related to the best approximation of $\alpha_{1}$; if $p_{n} / q_{n}$ is the $n$th convergent of $\alpha_{1}$, we have $\alpha_{1} \ldots \alpha_{n+1}=\left|q_{n} \alpha_{1}-p_{n}\right|$.

We finish by investigating what are all possible sequences $e_{n}$ for $x$ in $[0,1[$ :

Proposition 2. The sequences $e_{n}$ of Theorem 1 , for every $x \in[0,1$ [, are all the sequences of integers such that $0 \leq e_{n} \leq a_{n}$ and $e_{n} \neq a_{n}$ if $e_{n-1} \neq 0$.

Proof. We have $0 \leq e_{n} \leq a_{n}$; and, if $e_{n} \neq 0$, then $x_{n}<-\alpha_{n} \alpha_{n+1}(\leq$ if $n$ is even) and $e_{n} \geq 1$, hence $x_{n+1}>-1+\alpha_{n+1}$ ( $\geq$ if $n$ is even), which means exactly that $e_{n+1} \neq a_{n+1}$. Now, the map $f_{n}(y)=-\left(y+e_{n} \alpha_{n}\right) / \alpha_{n}$ is a decreasing linear map on the $a_{n}+1$ intervals of constancy of $e_{n}$, and so this map, which associates $x_{n+1}$ to $x_{n}$, is surjective on its intervals of continuity; hence the previous markovian condition on the sequence $e_{n}$ is the only one (see Figure 2).

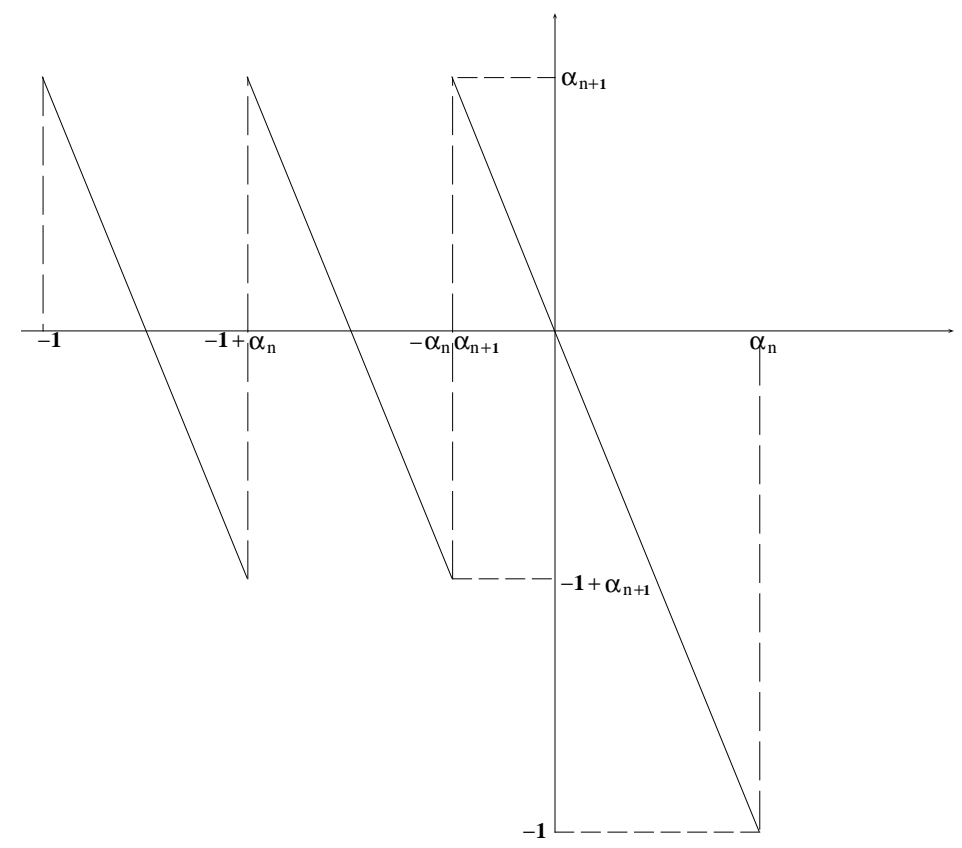

Fig. 2. Graph of the map $f_{n}$ in the case $a_{n}=2$ 
5. Additional remarks. Instead of using the symmetric Rauzy induction, one can use the original Rauzy induction, namely: the induction on the largest admissible interval containing 0 (see [RAU3] for a formal definition). This process leads to a different algorithm to compute the Sturmian sequence $U(\alpha, x)$ (see [ARN-FIS]). Such an algorithm is related to a numeration system which is dual to the one obtained in Theorem 1. For more details on the arithmetic and adic properties of this numeration system see [SID-VER].

With the previous notations, the expansion of the real number $x$ in this numeration system [SID-VER] is

$$
x=\sum_{n=0}^{\infty} \alpha_{1} \ldots \alpha_{n} b_{n}
$$

(with $0 \leq b_{n} \leq a_{n}$ and the Markov condition: $b_{n}=a_{n}$ implies $b_{n+1}=0$ ).

Given this expansion of $x$, the sequence $U(\alpha, x)$ can be explicitly computed using the sequences $\left(b_{n}\right),\left(a_{n}\right)$ and the two substitutions $\tau_{0}$ and $\tau_{1}$ defined by

$$
\tau_{0} 0=0, \tau_{0} 1=10, \tau_{1} 0=10, \tau_{1} 1=1 .
$$

\section{References}

[ALL] J. P. Allouche, Sur la complexité des suites infinies, Bull. Belg. Math. Soc. 1 (1994), 133-143.

[ARN-FIS] P. Arnoux and A. Fisher, The scenery flow for geometric structures on the torus, preprint, 1997.

[HED-MOR] G. A. Hedlund and M. Morse, Symbolic dynamics II. Sturmian trajectories, Amer. J. Math. 62 (1940), 1-42.

[ITO-YAS] S. Ito and S. Yasutomi, On continued fractions, substitutions and characteristic sequences $[n x+y]-[(n-1) x+y]$, Japan. J. Math. 16 (1990), 287-306.

[KEA] M. Keane, Sur les mesures quasi-ergodiques des translations irrationnelles, C. R. Acad. Sci. Paris 272 (1971), 54-55.

[KOM1] T. Komatsu, Results on fractional parts of linear functions of $n$ and applications to Beattie sequences, Ph.D. thesis, Macquarie University, 1994.

[KOM2] - On the characteristic word of the inhomogeneous Beatty sequence, Bull. Austral. Math. Soc. 51 (1995), 337-351.

[KOM3] - A certain power series associated with a Beatty sequence, Acta Arith. 76 (1996), 109-129.

[KOM4] - A certain power series and the inhomogeneous continued fraction expansions, J. Number Theory 59 (1996), 291-312.

[KOM5] - The fractional part of $n \theta+\phi$ and Beatty sequences, J. Théor. Nombres Bordeaux 7 (1995), 387-406.

[NIS-SHI-TAM] K. Nishioka, I. Shiokawa and J. Tamura, Arithmetical properties of a certain power series, J. Number Theory 42 (1992), 61-87. 
[OST] A. Ostrowski, Bemerkungen zur Theorie der Diophantischen Approximationen I, II, Abh. Math. Sem. Hamburg 1 (1922), 77-98, 250-251.

[RAU1] G. Rauzy, Une généralisation des développements en fractions continues, Sém. Delange-Pisot-Poitou 1976-1977, exp. 15.

[RAU2] -, Mots infinis en arithmétique, in: Lecture Notes in Comput. Sci. 192, Springer, 1985, 165-171.

[RAU3] - Échanges d'intervalles et transformations induites, Acta Arith. 34 (1979), 315-328.

[SID-VER] N. A. Sidorov and A. M. Vershik, Arithmetic expansions associated with rotations of the circle and continued fractions, St. Petersburg Math. J. 5 (1994), 1121-1136.

[SOS] V. T. Sós, On the distribution of the sequence no, Ann. Univ. Sci. Budapest. Eötvös Sect. Math. 1 (1958), 127-134.

Institut de Mathématiques de Luminy

Case 930

163 avenue de Luminy

F-13288 Marseille Cedex 9, France

E-mail: arnoux@iml.univ-mrs.fr ferenczi@iml.univ-mrs.fr hubert@iml.univ-mrs.fr 